\title{
Atypical presentation of Covid-19 in persons with spinal cord injury
}

\author{
Madeline A. Dicks ${ }^{1} \cdot$ Nathan D. Clements $^{1} \cdot$ C. R. Gibbons ${ }^{1} \cdot$ Monica Verduzco-Gutierrez $\mathbb{D}^{1} \cdot$ Michelle Trbovich $^{2}$
}

Received: 13 April 2020 / Revised: 1 May 2020 / Accepted: 1 May 2020

(c) International Spinal Cord Society 2020

\section{To the Editor:}

In light of the novel Coronavirus Disease (Covid-19) pandemic, we need to prepare to detect and treat this virus in vulnerable populations, including persons with spinal cord injury (SCI). We are writing this letter to heighten awareness amongst primary and acute care providers that individuals with SCI may not present with the typical Covid-19 symptoms of fever, cough and shortness of breath [1,2], and that as a result, their diagnosis may be delayed.

First, thermoregulatory dysfunction is a well-known sequela after spinal cord injury, due to disruption of neurologic signals to and from the hypothalamic temperature regulation center. This disruption results in subnormal $\left(<35.7^{\circ} \mathrm{C}\right)$ resting core body temperature, poikilothermia, and lack of the typical febrile response to infectious or inflammatory processes $>38^{\circ} \mathrm{C}$ as defined by the CDC and WHO $[1,3]$. More specifically, the impaired vasoconstriction and shivering, largely responsible for elevating core temperature during fever in persons without SCI, is proportional to the level of injury where persons with higher injury levels (i.e., tetraplegia) are more impaired than those with lower injuries (i.e., paraplegia) [4]. As with the elderly population, healthcare providers of persons with SCI need to have heightened awareness of their potential inability to mount a febrile response, which could delay diagnosis and treatment leading to poor outcomes [3].

In addition, respiratory dysfunction-a major cause of morbidity and mortality in SCI-leads to an increased risk for pulmonary infection and death in persons with SCI [4]. Vital capacity as low as 1-2 L (in non-ventilated persons with high tetraplegia) leads to insufficient ventilation, atelectasis and dyspnea on exertion. In addition, muscles of

Madeline A. Dicks

Dicks@uthscsa.edu

1 University of Texas Health Science Center at San Antonio, San Antonio, TX, USA

2 South Texas Veterans Health Care System, San Antonio, TX, USA expiration are often weakened leading to profound deleterious effects on peak cough flow (as low as $\sim 170 \mathrm{~L} / \mathrm{min}$ versus $>360 \mathrm{~L} / \mathrm{min}$ in able-bodied) that impairs secretion clearance [5]. In summary, SCI induced respiratory dysfunction increases susceptibility to viral pneumonia caused by Covid-19 and likely increased morbidity and mortality compared to their able-bodied counterparts. Furthermore, Veeravagu et al. found that persons with SCI are at greater risk of acute respiratory distress syndrome (ARDS) ( $95 \%$ incidence in critically ill persons with Covid-19) [6]. We suspect that, rather than cough, fever and shortness of breath (typical presenting symptoms in able-bodied persons), hypoxia ( $<90 \% \mathrm{SpO} 2)$, a worsened ability to clear secretions and tachypnea may be the initial symptoms in persons with SCI. Treatment of respiratory infections in persons with SCI includes early respiratory therapy involvement with aggressive pulmonary toilet including manually assisted coughing, mechanical assisted coughing (insufflation-exsufflation), chest percussion, vibrations and postural drainage [7]. Note that caution should be used with cough assist and manually assisted coughing to avoid exposure to aerosolized secretions, by ensuring appropriate PPE are worn. In appropriate settings and when available referral to spinal cord injury trained providers with experience in ventilation management in SCI may be indicated.

Given the physiologic differences from the able-bodied population, providers in the primary and acute care settings must be aware that patients with SCI may present without the typical presenting symptoms of fever, cough and shortness of breath in the setting of Covid-19. We suspect hypoxia may be the first objective symptom, so would recommend close oxygen monitoring in patients with suspected infection. If Covid-19 is confirmed in a person with SCI, we recommend aggressive pulmonary toilet to prevent pneumonia and monitoring in an ICU setting if beds are available. Through raising awareness of the atypical presentation of Covid-19 in persons with SCI, we hope that early detection and treatment may lessen its potential impact for significant morbidity and mortality in such vulnerable persons. 


\section{Compliance with ethical standards}

Conflict of interest The authors declare that they have no conflict of interest.

Publisher's note Springer Nature remains neutral with regard to jurisdictional claims in published maps and institutional affiliations.

\section{References}

1. WHO. Coronavirus disease 2019. Word Health Organization, 2020. https://doi.org/10.1001/jama.2020.2633.

2. National Center for Immunization and Respiratory Diseases (NCIRD) D of VD. Interim Clinical Guidance for Management of Patients with Confirmed Coronavirus Disease (COVID-19). Centers Dis Control Prev. 2020. https://doi.org/10.1164/rccm.201908-1581ST.
3. Trbovich M, Li C, Lee S. Does the CDC definition of fever accurately predict inflammation and infection in person with SCI? Top Spinal Cord Inj Rehabil. 2016. https://doi.org/10.1210/ sci2016-00049.

4. Schmidt KD, Chan CW. Thermoregulation and fever in normal persons and in those with spinal cord injuries. Mayo Clin Proc. 1992;67:469-75.

5. Cardozo CP. Respiratory complications of spinal cord injury. J Spinal Cord Med. 2007. https://doi.org/10.1080/10790268.2007. 11753945.

6. Veeravagu A, Jiang B, Rincon F, Maltenfort M, Jallo J, Ratliff JK. Acute respiratory distress syndrome and acute lung injury in patients with vertebral column fracture(s) and spinal cord injury: a nationwide inpatient sample study. Spinal Cord. 2013;51:461-5. https://doi.org/10.1038/sc.2013.16. Epub 2013 Mar 12.

7. Vázquez G, Sedes R, Fariña M, Marqués M, Velasco F. Respiratory management in the patient with spinal cord injury. Biomed Res Int. 2013. https://doi.org/10.1155/2013/168757. 